\title{
Cathode Ion Bombardment in RF Photoguns
}

\author{
E. Pozdeyev, D. Kayran, V. Litvinenko
}

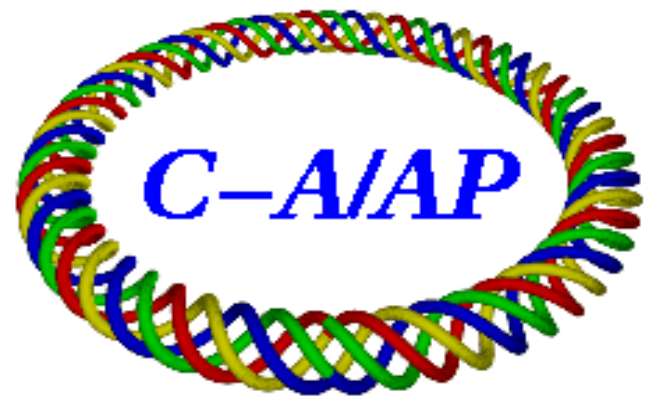

Collider-Accelerator Department Brookhaven National Laboratory Upton, NY 11973

Notice: This document has been authorized by employees of Brookhaven Science Associates, LLC under Contract No. DE-AC02-98CH10886 with the U.S. Department of Energy. The United States Government retains a nonexclusive, paid-up, irrevocable, world-wide license to publish or reproduce the published form of this document, or allow others to do so, for United States Government purposes. 


\title{
CATHODE ION BOMBARDMENT IN RF PHOTOGUNS
}

\author{
E. Pozdeyev,* D. Kayran, and V. Litvinenko \\ Brookhaven National Laboratory, Upton, NY 11973-5000
}

(Dated: September 3, 2008)

\begin{abstract}
In this paper, we use the method of rapid oscillating field to solve the equation of ion motion in an RF gun. We apply the method to the BNL 1/2-cell SRF photogun and demonstrate that a significant portion of ions produced in the gun can reach the cathode if no special precautions are taken. Also, the paper proposes a simple mitigation recipe that can reduce the rate of ion bombardment.
\end{abstract}

PACS numbers: 29.25. Bx,85.60.Ha

\section{INTRODUCTION}

Radiofrequency (RF) photoguns with negative electron affinity (NEA) GaAs cathodes have a potential to deliver high peak current, high quality, high average intensity electron beams. Such beams are required for linac and energy recovery linac (ERL) driven light sources, free electron lasers, and nuclear and high energy physics experiments. Additionally, nuclear and high energy physics experiments require a high degree of polarization. NEA GaAs cathodes stand out from other cathode options because of their ability to produce polarized beams. Also, GaAs cathodes can have quantum efficiency (QE) as high as $10 \%$ (unpolarized). Several efforts to explore feasibility of using GaAs cathodes in RF guns have been undertaken (see, for example [1],[2],[3]).

GaAs cathodes are used extensively in static voltage (DC) photoinjectors, polarized and not polarized. The operational experience with DC guns has demonstrated that ion backbombardment is the major cause of degradation of QE of GaAs cathodes. Numerical simulations described in [2], [4] have shown that ion bombardment can happen in RF guns too, possibly limiting the lifetime of cathodes. Although numerical simulations are useful for a specific gun, they give little insight in the motion of ions in general and are hard to extrapolate to other guns if scaling laws are not known.

In this paper, we apply the method of rapidly oscillating field to analyze the motion of ions in RF guns. The method was originally proposed by Kapitza in 1951 [5] and applied by Gaponov and Miller to charged particles in oscillating electro-magnetic field in 1958 [6]. We briefly describe the method in section II, emphasizing the importance of the initial conditions in section IIB. Using the method, we examine the ion motion in axially symmetric guns in section III and apply the method to the BNL 1/2-cell SRF gun in section IV.

Note that electron backbombardment and multipackting can affect the cathode life time in RF guns as well. However, we will not discuss electron backbombardment in this paper.

*pozdeyev@bnl.gov

\section{MOTION OF IONS IN RAPIDLY OSCILLATING RF FIELD}

\section{A. Effective potential energy of an ion in RF field}

The motion of an ion with the mass $m$ and charge $q$ in an RF gun is described by the equation of motion (in the centimeter-gram-second system of units):

$$
m \ddot{\mathbf{r}}=q \mathbf{E}+\frac{q}{c} \mathbf{v} \times \mathbf{B},
$$

where $\mathbf{E}$ and $\mathbf{B}$ are the electric and magnetic fields respectively. $c$ is the speed of light. An accurate analytical solution of (1) is impossible in the general case. However, equation (1) can be solved iteratively using the method of rapidly oscillating field. According to the method, the motion of the ion can be described as a superposition of the fast oscillating term a and the term describing the ion motion averaged over the fast oscillations $\mathbf{x}(t)=\overline{\mathbf{r}(t)}$ :

$$
\mathbf{r}=\mathbf{x}+\mathbf{a}
$$

The method is applicable if the amplitude of fast oscillations, $|\mathbf{a}|$, is small comparatively to the characteristic size of inhomogenuity of the RF field, $L$ :

$$
\frac{|\mathbf{a}|}{L} \ll 1
$$

This condition can be also written in the equivalent form:

$$
|(\mathbf{a} \cdot \nabla) \mathbf{E}| \ll|\mathbf{E}| .
$$

Substituting (2) into (1) and leaving only terms of the first order in $|\mathbf{a}| / L$, one obtains the following equation:

$$
m(\ddot{\mathbf{x}}+\ddot{\mathbf{a}})=q \mathbf{E}+q(\mathbf{a} \cdot \nabla) \mathbf{E}+\frac{q}{c} \mathbf{v} \times \mathbf{B} .
$$

The electric and magnetic fields on the right hand side of (5) are taken at $\mathbf{x}$. Note that the magnetic field term $\mathbf{v} \times \mathbf{B} / c$ is of the order of $|\mathbf{a}| / L$, same as $(\mathbf{a} \cdot \nabla) \mathbf{E}$, and is much smaller than $\mathbf{E}$ :

$$
\left|\frac{\mathbf{v} \times \mathbf{B}}{c}\right| \sim\left|\frac{\mathbf{a}}{\lambda} \times(\lambda \nabla \times \mathbf{E})\right| \sim \frac{|\mathbf{a}|}{L}|\mathbf{E}| \ll|\mathbf{E}|,
$$

where $\lambda$ is the RF wavelength divided by $2 \pi$. 
Equation (5) can be solved by iterations. The $0^{\text {th }}$-order equation is obtained by using only the term $q \mathbf{E}$ on the right-hand side and omitting the other terms. Because $q \mathbf{E}$ is a fast oscillating term, the second derivative $\ddot{\mathbf{x}}$ can be also neglected. If the RF electric field has the following form

$$
\mathbf{E}=\mathcal{E}(\mathbf{r}) \cos (\omega t+\psi)
$$

where $\omega t+\psi$ is the RF phase, the equation for $\mathbf{a}$ is written as

$$
m \ddot{\mathbf{a}}=q \mathcal{E} \cos (\omega t+\psi) .
$$

A particular solution of (8) is

$$
\mathbf{a}=-\lambda^{2} \frac{q \mathcal{E} \cos (\omega t+\psi)}{m c^{2}}, \quad \dot{\mathbf{a}}=\star c \frac{q \mathcal{E} \sin (\omega t+\psi)}{m c^{2}} .
$$

The first-order approximation is obtained by substituting (9) into (5) and averaging over the fast oscillations. This yields the equation for the averaged motion:

$$
\ddot{\mathbf{x}}=-\frac{\lambda^{2} c^{2}}{4}\left(\frac{q}{m c^{2}}\right)^{2} \nabla \mathcal{E}^{2} .
$$

Equation (10) shows that the effect of the RF field averaged over the fast oscillations can be described by the effective potential energy

$$
U_{e}=\frac{m c^{2}}{4}\left(\frac{\lambda q \mathcal{E}}{m c^{2}}\right)^{2}=\frac{Z^{2}}{A} \frac{m_{u} c^{2}}{4}\left(\frac{\lambda e \mathcal{E}}{m_{u} c^{2}}\right)^{2},
$$

where $Z$ and $A$ are the ion charge and mass states respectively, $e$ is the elementary charge, and $m_{u} c^{2}$ is the energy equivalent of the atomic mass unit, $931.5 \mathrm{MeV}$. Note that the effective potential energy is numerically equal to the average kinetic energy of the fast oscillations:

$$
U_{e}=m \frac{\overline{\dot{\mathbf{a}}^{2}}}{2} .
$$

The Lagrange function of the averaged motion is

$$
L=\frac{m}{2} \dot{\mathbf{x}}^{2}-U_{e}=T_{e}-U_{e}
$$

where we introduced the effective kinetic energy $T_{e}=$ $m \dot{\mathbf{x}} / 2$.

If the amplitude of the electric field does not depend on time, the total effective energy is the integral of (10):

$$
\frac{m}{2} \dot{\mathbf{x}}^{2}+U_{e}=T_{e}+U_{e}=\text { const. }
$$

\section{B. Initial conditions and effective kinetic energy of ions}

In treating the ion motion, we will assume that ions are produced only in collisions of the electron beam with the residual gas and neglect other sources of ionization. The initial velocity of an ion is given by

$$
\dot{\mathbf{r}}_{0}=\dot{\mathbf{x}}_{0}+\dot{\mathbf{a}}_{0}=\dot{\mathbf{x}}_{0}+\lambda c \frac{q \mathcal{E} \sin \left(\phi_{0}\right)}{m c^{2}},
$$

where $\phi_{0}$ is the RF phase at which ionization happens. The energy transferred in the ionization process is mostly absorbed by knocked out electrons [7]. This means that the energy of ions is close to the average thermal energy, which is approximately $0.04 \mathrm{eV}$ at room temperature. It will be demonstrated later in this paper that both the kinetic energy of the fast oscillations and the kinetic energy of the averaged motion are typically much larger than the thermal energy. Therefore, it can be assumed that ions originate at rest. Assuming $\dot{\mathbf{r}}_{0}=0$, one finds $\dot{\mathbf{x}}_{0}$ :

$$
\dot{\mathbf{x}}_{0}=-\lambda c \frac{q \mathcal{E} \sin \left(\phi_{0}\right)}{m c^{2}} .
$$

The associated effective kinetic energy is given by:

$$
\begin{array}{r}
T_{e 0}=m \frac{\dot{\mathbf{x}}_{0}^{2}}{2}=\frac{Z^{2}}{A} \frac{m_{u} c^{2}}{2}\left(\frac{\lambda e \mathcal{E}}{m_{u} c^{2}}\right)^{2} \sin ^{2}\left(\phi_{0}\right) \\
=2 U_{e} \sin ^{2}\left(\phi_{0}\right) .
\end{array}
$$

Both $U_{e}$ and $T_{e 0}$ are quadratic functions of the charge and, therefore, do not depend on the sign of the ion charge. The initial velocity $\dot{\mathbf{x}}_{0}$ is a linear function of the ion charge and has opposite signs for positive and negative ions. In this paper, we will consider only positively charged ions, assuming that the number of negative ions is small.

\section{APPLICATION OF THE METHOD TO AXIALLY SYMMETRIC GEOMETRY}

Typically, an RF gun consist of one or more axially symmetric cavities with the cathode situated in the upstream wall of the first cavity. The electron beam is produced at the cathode and is accelerated by the RF field along the gun axis. Power and higher order couplers can violate the gun symmetry and induce multipole fields. The field of these multipoles is typically small close to the gun axis and can be neglected. Therefore, we will assume axial symmetry and choose the $z$-axis directed along the gun axis with the coordinate $z=0$ corresponding to the cathode.

\section{A. Motion of ions on the gun axis, 1D case}

The axial symmetry of the problem requires the electric field have only the $z$-component and the magnetic field be zero on the gun axis. Thus, the ion motion on the gun axis is one-dimensional. If the potential energy and the initial conditions are known, solving the onedimensional problem of the ion motion is trivial. The 
condition that the total energy has to be larger than the potential energy defines areas where the ion motion is possible. The detailed solution $\mathbf{x}(t)$ of $(10)$ can be obtained by trivial integration of the integral of motion (14).

Because we assumed that ions are produced only by the beam, the ionization phase $\phi_{0}$ can expressed as a function of $z$. For a given $z, \phi_{0}(z)$ is equal to the RF phase at which electron bunches pass $z$. The electric field $\mathcal{E}(\mathbf{r})$ and the function $\phi_{0}(z)$ uniquely define the initial conditions and allow one to solve the 1D problem of the ion motion as described in the previous paragraph.

Note that the dependence of the initial drift velocity $\dot{\mathbf{x}}_{0}$ on the accelerating phase can be employed to suppress ion bombardment in a single-cell gun. If the electric field is given by (7), the force acting on electrons is $\mathbf{F}_{\text {acc }}=-e \mathcal{E}(\mathbf{r}) \cos (\omega t+\psi)$. Assuming that this force accelerates the beam in the phase range from $-\pi / 2$ to $\pi / 2$, one can easily see that $\dot{\mathbf{x}}_{0}$ points in the direction of acceleration if the phase $\phi_{0}$ is between 0 and $\pi / 2$ and in the opposite direction if $\phi_{0}$ is between $-\pi / 2$ and 0 . Thus, the initial drift velocity $\dot{\mathbf{x}}_{0}$ of all ions will point out of the gun if the RF phase changes between 0 and $\pi / 2$ (plus an integer number of full RF cycles) during acceleration of electron bunches. Ions with the drift velocity pointing out of the gun and the total effective energy greater than the effective potential energy for all $z$ greater than the ionization coordinate will leave the gun. Only a small portion of ions originating close to the cathode still will be able to strike the cathode. Because a positively charged ion is accelerated towards the cathode right after ionization, it can reach the cathode during the first RF cycle after the ionization. If the ion does not strike the cathode on the first RF cycle, it will drift away from the cathode. The distance from which the ion can reach the cathode depends on the RF phase at which the beam is produced. However, this distance cannot be larger than the double amplitude of the fast oscillations near the cathode.

In the end, note that the accelerating phase range from 0 to $\pi / 2$, that is, when the accelerating field strength decreases, might not be optimal from the emittance and energy spread point of view. Also, the suggested method will not work in multi-cell guns because the accelerating phase cannot be limited to the range between 0 and $\pi / 2$. In this case, however, cathode biasing can be used. As shown later in Section IV, a bias voltage of a few hundred volts might be sufficient to repel a large portion of ions from the cathode.

\section{B. Motion of ions off axis}

The radial beam size and the cathode are typically much smaller than $\lambda$ in RF guns. Therefore, we will consider only ion trajectories with a small radial deviation from the gun axis comparatively to $\lambda$. The electric field off the gun axis can be expressed via the electric field on the gun axis and its derivatives with respect to $z$. Using the notation

$$
\mathcal{E}_{a}(z)=\mathcal{E}_{z}(z, r=0),
$$

we can write the electric field $\mathcal{E}(z, r)$ to the second order in $r$ as

$$
\begin{aligned}
& \mathcal{E}_{z}(z, r)=\mathcal{E}_{a}-\frac{1}{4}\left(\mathcal{E}_{a}^{\prime \prime}+\frac{\mathcal{E}_{a}}{\lambda^{2}}\right) r^{2}+\ldots \\
& \mathcal{E}_{r}(z, r)=-\frac{\mathcal{E}_{a}^{\prime}}{2} r+\ldots
\end{aligned}
$$

where' stands for $\mathrm{d} / \mathrm{dz}$.

According to (11), the effective potential energy of the $\mathrm{RF}$ field is proportional to the amplitude of the electric field squared. $\mathcal{E}^{2}$ can be written off axis as:

$$
\begin{aligned}
\mathcal{E}^{2}=\mathcal{E}_{z}^{2}+\mathcal{E}_{r}^{2} & =\left(\mathcal{E}_{a}+\delta \mathcal{E}_{z}\right)^{2}+\mathcal{E}_{r}^{2} \\
& \approx \mathcal{E}_{a}^{2}+2 \mathcal{E}_{a} \delta \mathcal{E}_{z}+\mathcal{E}_{r}^{2} .
\end{aligned}
$$

Equations (19a-20) and (11) yield the effective potential energy to the second order in $r$ as

$$
\begin{aligned}
U_{e} & =\frac{Z^{2}}{A} \frac{m_{u} c^{2}}{4}\left(\frac{\lambda e}{m_{u} c^{2}}\right)^{2} \\
& \times\left(\mathcal{E}_{a}^{2}-\frac{\mathcal{E}_{a}}{2}\left(\mathcal{E}_{a}^{\prime \prime}+\frac{\mathcal{E}_{a}}{\lambda^{2}}\right) r^{2}+\frac{\left(\mathcal{E}_{a}^{\prime}\right)^{2}}{4} r^{2}\right) .
\end{aligned}
$$

The radial motion of an ion averaged over fast oscillations is described by the Lagrange equation

$$
\frac{\mathrm{d}}{\mathrm{d} t} \frac{\partial L}{\partial \dot{r}}=\frac{\partial L}{\partial r}
$$

with the Lagrangian $L$ given by (13). Under the assumption that ions originate at rest, the angular component of the ion velocity and the corresponding angular momentum can be neglected and the equation for the average ion radius can be written as

$$
m \ddot{r}=-\frac{\partial U_{e}}{\partial r}=F_{r},
$$

with $F_{r}$ given by

$$
\begin{aligned}
F_{r} & =\frac{Z^{2}}{A} \frac{m_{u} c^{2}}{4}\left(\frac{\lambda e}{m_{u} c^{2}}\right)^{2} \\
& \times\left(\mathcal{E}_{a}\left(\mathcal{E}_{a}^{\prime \prime}+\frac{\mathcal{E}_{a}}{\lambda^{2}}\right)-\frac{\left(\mathcal{E}_{a}^{\prime}\right)^{2}}{2}\right) r .
\end{aligned}
$$

Equation (23) has to be solved simultaneously with the Lagrange equation for $z$. However, because we are interested only in ion trajectories with a small deviation from the gun axis, we can neglect the effect of the radial motion on the axial motion. That is, we can solve (23) assuming that the axial motion does not depend on $r$ and use the value of $\dot{x}_{z}$ on the gun axis.

Equation (23) has to be solved numerically in the general case. A solution of (23) can be also found by iterations. If the trajectory radius changes little, we can limit 
the solution to the first iteration. That is, we write the ion trajectory radius as $r=r_{0}+r_{1}$, where $r_{0}$ is the initial radius, and the equation for the first iteration $r_{1}$ as

$$
m \ddot{r}_{1}=F_{r}\left(r_{0}\right) .
$$

The standard method of variation of constants yields the solution of (25)

$$
r_{1}(t)=\int_{0}^{t} \frac{F_{r}}{m}(t-\tau) \mathrm{d} \tau+\dot{x}_{r 0} t,
$$

where $\dot{x}_{r 0}$ is the $r$-projection of $\dot{\mathbf{x}}_{0}$. It is convenient to change the independent variable from $t$ to $z$ according to

$$
t=\int_{z_{0}}^{z} \frac{\mathrm{d} \xi}{\dot{x}_{z}(\xi)},
$$

where $\dot{x}_{z}$ is the $z$-projection of $\dot{\mathbf{x}}$ and $z_{0}$ denotes the ionization coordinate. Note that $\dot{x}_{z}$ depends on $z_{0}$ as a parameter. The time differential is given by $\mathrm{d} t=\mathrm{d} z / \dot{x}_{z}$. Thus, $r_{1}(z)$ can be written as

$$
\begin{aligned}
r_{1}(z)=\int_{z}^{z_{0}} & \frac{F_{r}\left(\xi^{\prime}\right) \mathrm{d} \xi^{\prime}}{m \dot{x}_{z}\left(\xi^{\prime}\right)} \int_{z}^{\xi^{\prime}} \frac{\mathrm{d} \xi^{\prime \prime}}{\dot{x}_{z}\left(\xi^{\prime \prime}\right)} \\
& -\left(\frac{\mathrm{d} r}{\mathrm{~d} z}\right)_{0} \int_{z}^{z_{0}} \frac{\dot{x}_{z 0} \mathrm{~d} \xi}{\dot{x}_{z}(\xi)} .
\end{aligned}
$$

Using equations (14), (16-17), (24), and (28) and taking into account $\dot{x}_{r} \ll \dot{x}_{z}$, one obtains the radial deviation of the ion trajectory at the cathode, $r_{1 c}=r_{1}(0)$, normalized to $r_{0}$ :

$$
\begin{aligned}
& \frac{r_{1 c}}{r_{0}}= \int_{0}^{z_{0}} \frac{\left(\mathcal{E}_{a}\left(\mathcal{E}_{a}^{\prime \prime}+\frac{\mathcal{E}_{a}}{\lambda^{2}}\right)-\frac{\mathcal{E}_{a}^{\prime 2}}{2}\right) \mathrm{d} \xi^{\prime}}{2 \sqrt{\mathcal{E}_{a 0}^{2}\left(1+2 \sin ^{2}\left(\phi\left(z_{0}\right)\right)\right)-\mathcal{E}_{a}^{2}\left(\xi^{\prime}\right)}} \\
& \times \int_{0}^{\xi^{\prime}} \frac{\mathrm{d} \xi^{\prime \prime}}{\sqrt{\mathcal{E}_{a 0}^{2}\left(1+2 \sin ^{2}\left(\phi\left(z_{0}\right)\right)\right)-\mathcal{E}_{a}^{2}\left(\xi^{\prime \prime}\right)}} \\
&+\frac{\mathcal{E}_{a 0}^{\prime}}{2 \mathcal{E}_{a 0}} \int_{0}^{z_{0}} \sqrt{\frac{2 \mathcal{E}_{a 0}^{2} \sin ^{2}\left(\phi\left(z_{0}\right)\right)}{\mathcal{E}_{a 0}^{2}\left(1+2 \sin ^{2}\left(\phi\left(z_{0}\right)\right)\right)-\mathcal{E}_{a}^{2}(\xi)}} \mathrm{d} \xi
\end{aligned}
$$

where $\mathcal{E}_{a 0}$ denotes $\mathcal{E}_{a}\left(z_{0}\right)$. Equation (29) is valid only if the inequality $r_{1}(z) / r_{0} \ll 1$ is satisfied for all $z$.

\section{Effect of the electron beam on the ion motion}

\section{Effect on the axial motion}

The axial beam field is induced by features that break the continuous transitional symmetry along the gun axis, including the cathode, variation of the beam velocity, variation of the beam size, and the transition from the gun cavity to the transfer beam line. The effect of the electron beam on the axial motion of ions is hard to calculate accurately. First, neither non-relativistic nor ultrarelativistic limit can be applied to simplify calculation of the beam field because the beam energy changes from almost zero to a few MeV's during acceleration. Second, calculation of the field of image charges is complicated because of non-trivial geometry of the boundaries. However, we can crudely estimate each of these effects and compare the induced potential difference to the effective kinetic energy of the averaged motion.

The potential difference induced by the beam size variation and the transition from the gun to the transfer line can be crudely estimated as [7]

$$
\delta U_{b}=\frac{2 I}{c} \ln \left(\frac{a_{1} b_{2}}{a_{2} b_{1}}\right),
$$

where $a_{1,2}$ and $b_{1,2}$ are respectively the beam size and the vacuum pipe radius at two different locations. Neglecting the beam size variation and assuming that the vacuum pipe radius is five times smaller than the gun cavity radius $\left(b_{1} / b_{2} \sim 5\right)$, we obtain $\left|\delta U_{b}\right|=0.08 \mathrm{~V} / \mathrm{mA}$. This number should be approximately the same for all $\mathrm{RF}$ guns because of the logarithmic dependence on the pipe radius .

To crudely estimate the effect of image charges at the cathode and the effect of variation of the beam velocity one can assume that the beam is nonrelativistic and use a static field solver such as Poisson [8] or similar. The CW beam has to be represented by a DC beam with the same current and the charge density given by

$$
\rho(z)=\frac{I}{v(z) S},
$$

where $v$ is the beam velocity and $S$ is the beam cross section area. Simulations of the beam field in the BNL 1/2cell SRF gun presented in Section IV yielded the voltage $\left|\delta U_{c}\right| \approx 0.2 \mathrm{~V} / \mathrm{mA}$.

The estimated potential energy difference has to be compared to the typical value of the effective kinetic energy of the averaged motion. If they are comparable, the beam induced potential has to be simulated accurately and added to the potential energy $U_{e}$ given by (11). If the estimated potential drop is small comparatively to $T_{e}$ for most of ions, the beam effect on the axial motion of ions can be neglected. Later in Section IV, we will demonstrate that the beam effect on the axial motion is small in the BNL SRF gun up to a beam current of a few hundred milliamperes.

\section{Effect on the radial motion}

According to the standard approach described elsewhere (for example, [7]), the transverse motion of ions in the $\mathrm{CW}$ beam is equivalent to the motion in a focusing channel composed of focusing lenses and drifts. Treatment of such motion involves the standard matrix analysis and is straight forward.

If the beam is the only source of transverse focusing, the periodic oscillatory motion of ions can be characterized by a phase advance per ion-bunch collision. For a 
round relativistic beam, the phase advance is

$$
\mu=\arccos \left(1-\frac{Z N_{e} r_{p} c T_{b}}{A a^{2}}\right)
$$

where $N_{e}$ is the number of electrons per bunch, $T_{b}$ is the time interval between electron bunches, $r_{p}$ is the classical proton radius, $a$ is the beam radius. If the phase advance exceeds $\pi$, the ion motion becomes unstable. The current at which ions become unstable is given by

$$
I_{\mathrm{th}}=\frac{2 A a^{2} e}{Z r_{p} c} f_{b}^{2}
$$

where $f_{b}$ is the bunch repetition rate $1 / T_{b}$. If the ion motion is unstable, the amplitude of ion oscillations grows exponentially with the number of collision until it exceeds the beam size. If the beam current is smaller than the threshold current, the ions are confined transversely within the beam.

In principle, one can calculate ion trajectories to almost any degree of accuracy considering individual collisions with electron bunches and solving (23) between the collisions. However, we would like to emphasize that in order to estimate the rate of cathode ion bombardment it is sufficient to find out which ions can reach the cathode without knowing exact details of their trajectories. Below the threshold current (33), the beam focuses ions towards the gun axis and brings more ions to the cathode. Thus, one can estimate the rate of cathode ion bombardment without the beam and use this number as the lower boundary estimate for the ion bombardment with the beam. Above the stability threshold, a similar approach can be applied to ions whose amplitude of oscillations does not grow substantially.

\section{BNL 1/2-CELL SRF GUN}

Brookhaven National Laboratory and Advanced Energy Systems, Inc. are jointly developing a $1 / 2$-cell superconducting radio-frequency (SRF) photogun [9]. The gun will serve as an injector for the BNL R\&D ERL [10] and a test bed for different types of photocathodes. Figure 1 shows the SuperFish [8] model of the gun. Table I lists main gun parameters. Figure 2 shows the accelerating electric field on the gun axis calculated by SuperFish.

The operational residual gas pressure in the gun is expected to be of the order of $10^{-11}$ Torr or lower. At this pressure, the residual gas will mostly consist of hydrogen. Using (9) and the gun parameters listed in Table I, one can easily estimate that the amplitude of oscillations of $\mathrm{H}_{2}^{+}$ions in the gun is smaller than $60 \mu \mathrm{m}$. This is much smaller than the characteristic spatial scale of field inhomogenuity. Thus, the method of rapid oscillating field can be applied to the BNL gun.

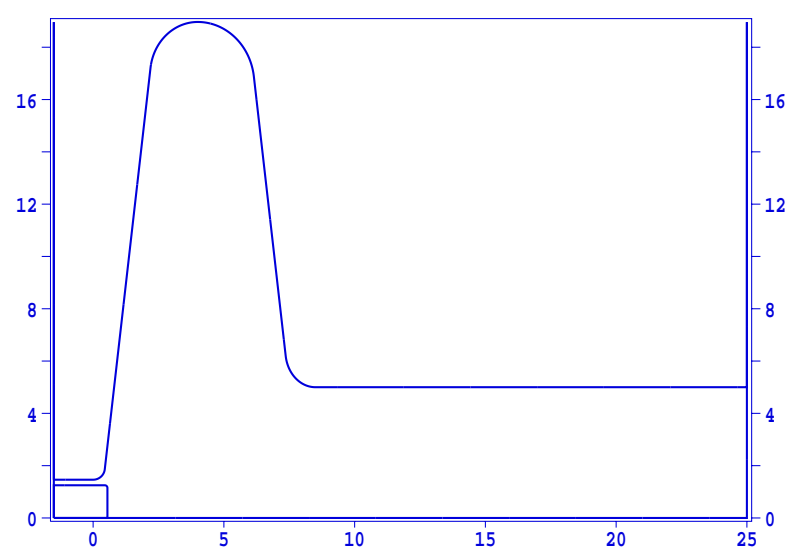

FIG. 1: SuperFish model of the BNL 1/2-cell SRF gun. The horizontal axis corresponds to the gun axis. The vertical axis is the radius. All dimensions are in centimeters. The cathode is at the low left corner of the figure.

TABLE I: Parameters of the BNL 1/2-cell SRF Gun. Nominal values are shown. Also, these numbers are used in the paper. The numbers in parentheses show values after a possible laser upgrade.

\begin{tabular}{ll}
\hline \hline Parameter & Value \\
\hline Beam Energy $(\mathrm{MeV})$ & 2.0 \\
$\mathcal{E}_{\text {max }}(\mathrm{MeV} / \mathrm{m})$ & 29 \\
$F_{\mathrm{RF}}(\mathrm{MHz})$ & 703.75 \\
$F_{\text {bunch }}(\mathrm{MHz})$ & $9.38(352)$ \\
$q_{\text {bunch }}(\mathrm{nC})$ & $0.7-5$ \\
$I_{\text {beam }}(\mathrm{mA})$ & $7-50(500)$ \\
\hline \hline
\end{tabular}

\section{A. Motion of ions on the gun axis}

The electric field calculated by SuperFish (Fig. 2) and equation (11) yield the effective potential energy. The program Parmela [11] was used to calculate the RF phase at which electron bunches pass a given coordinate $z$. It was assumed that bunches were short and the ionization phase corresponded to the middle of the bunch. Figure 3 shows the ionization phase $\phi_{0}$ as a function of $z$. The initial beam phase in this simulation was chosen to minimize the beam emittance. Equation (17) and $\phi_{0}(z)$ yield the initial effective kinetic energy $T_{e 0}$ as a function of the ionization coordinate.

Figure 4 shows the effective potential energy of $\mathrm{H}_{2}^{+}$ ions on the gun axis. Also, Figure 4 shows the total effective energy of $\mathrm{H}_{2}^{+}$ions as a function of the ionization coordinate. The total energy curve is divided into two branches shown by different colors: ions whose velocity $\dot{\mathbf{x}}_{0}$ points towards the cathode belong to the green branch $(z<4.5 \mathrm{~cm})$ while ions whose velocity points out of the gun belong to the blue branch $(z>4.5 \mathrm{~cm})$. At $z \approx 4.5$ $\mathrm{cm}$, the total effective energy is equal to the potential 


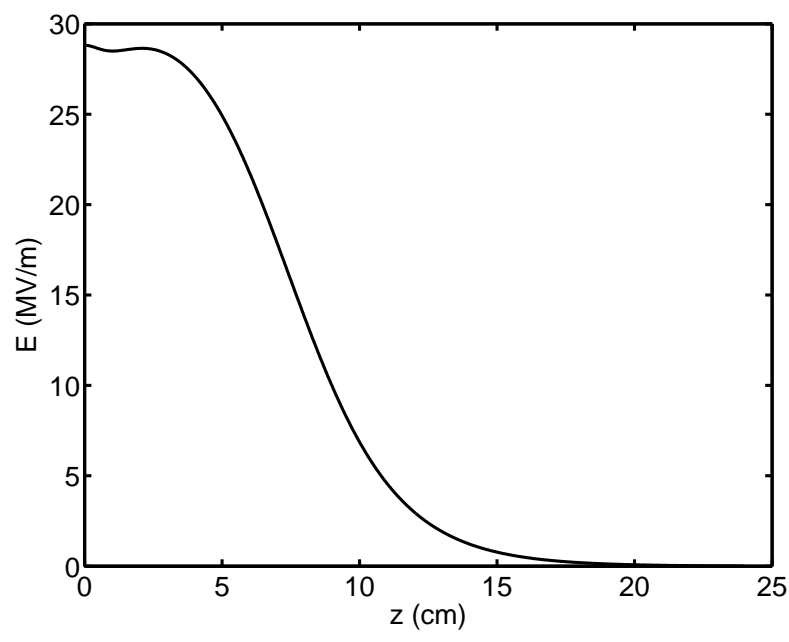

FIG. 2: Longitudinal electric field on the gun axis.

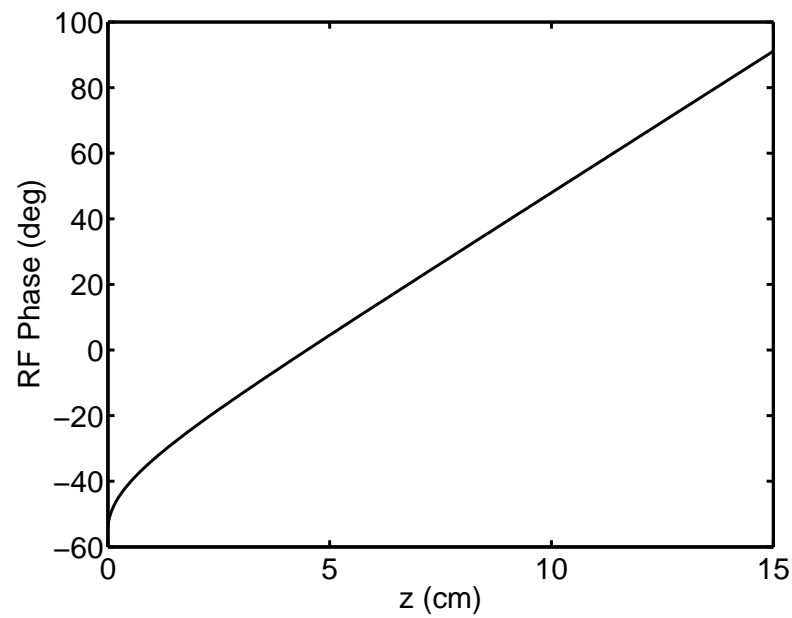

FIG. 3: RF phase at which electron bunches pass a given coordinate $z$.

energy that corresponds to $\dot{\mathbf{x}}_{0}=0$.

The total effective energy of ions originating at $z<3.4$ $\mathrm{cm}$ is larger than the effective potential energy at the cathode. Also, the velocity $\dot{\mathbf{x}}_{0}$ of these ions is directed towards the cathode. Thus, ions originating at $z<3.4$ $\mathrm{cm}$ will reach the cathode. The effective kinetic energy $T_{e}$ of ions bombarding the cathode is smaller than $700 \mathrm{eV}$. All other ions produced at $z \geq 3.4 \mathrm{~cm}$ will be expelled from the gun.

As discussed in Section III C 1, interaction of the beam field with the beam enclosure, image charges on the cathode, and the variation of the beam velocity can induce the longitudinal electric field on the gun axis.

Equation (30) gives an estimate of the potential drop caused by the transition from the gun cavity to the transfer line. The ratio of the gun cavity diameter to the transfer line diameter is approximately five to one. With this number, equation (30) yields a potential difference of $0.08 \mathrm{~V} / \mathrm{mA}$.

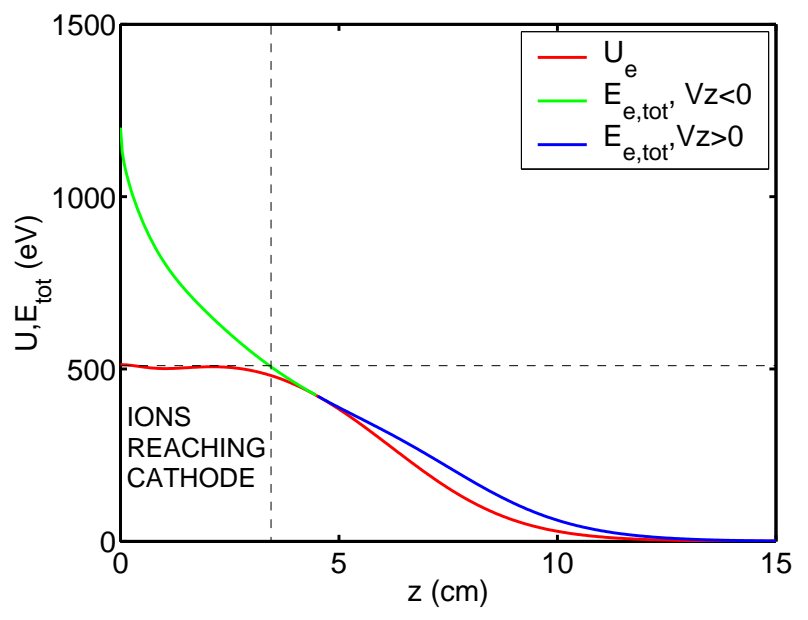

FIG. 4: The effective potential energy (red curve) and total effective energy (green and blue curves) of $\mathrm{H}_{2}^{+}$ions in the BNL $1 / 2$-cell SRF Gun. The green and blue curves show the total energy of ions with the effective velocity $\dot{\mathbf{x}}_{0}$ pointing towards the cathode and from the cathode respectively. Ions whose initial velocity is directed towards the cathode and total energy is higher than the maximum potential energy will reach the cathode.

To calculate the potential produced by image charges at the cathode we used the code Poisson [8] and the gun model shown in Fig. 1. The beam was modeled by a number of uniformly charged cylinders with the radius equal to the local beam radius. The radius of the beam was linearly changing from $2.5 \mathrm{~mm}$ at the cathode to approx $3.5 \mathrm{~mm}$ at $z=10 \mathrm{~cm}$. The charge density of each cylinder was given by (31). The simulated potential well had a minimum located at $z \approx 2 \mathrm{~cm}$. The depth of the well was approximately equal to $0.2 \mathrm{~V} / \mathrm{mA}$.

Considering that the effective kinetic energy of most of ions that can reach the cathode is above $100 \mathrm{eV}$, we can neglect the effect of the beam field on the ion axial motion up to a beam current of a few hundred milliamperes that is much higher than the nominal beam current at 9.38 $\mathrm{MHz}$ bunch repetition rate (Table I).

At the end, we demonstrate that ions meet only a few bunches on their way to the cathode. The number of ion-bunch collisions is given by

$$
n_{c}=\frac{1}{2 \pi h \lambda} \int_{0}^{z_{0}} \sqrt{\frac{A m_{u} c^{2}}{2 T_{e}(\xi)}} \mathrm{d} \xi
$$

where $h$ is the harmonic number. Figure 5 shows the number of ion-bunch collisions for the $9.38 \mathrm{MHz}$ operational mode $(h=75)$ as a function of the ionization coordinate $z_{0}$. (The collision of an ion with the bunch which produces the ion is not included.) According to the result, ions originating between 0 and $1.5 \mathrm{~cm}$ do not collide with the beam and, thus, are not affected by the beam. 


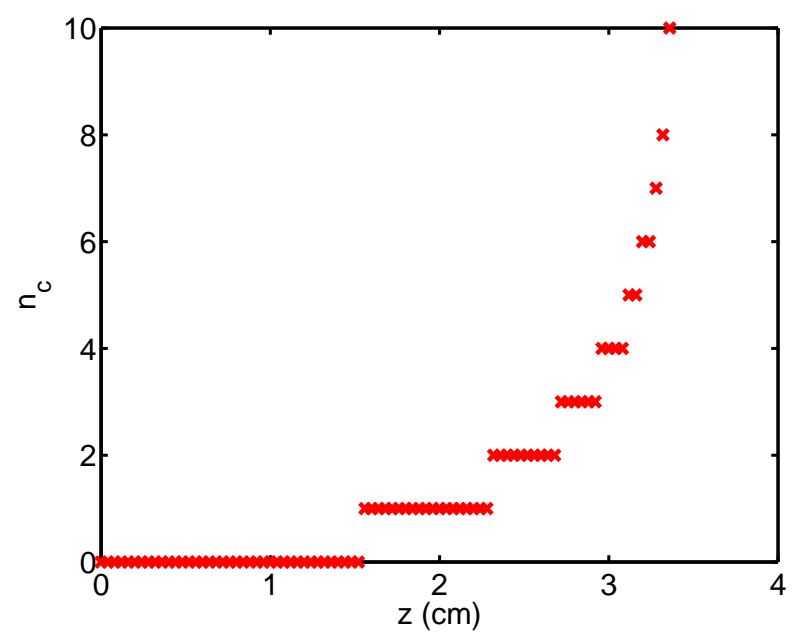

FIG. 5: Number of ion-bunch collisions as a function of the ionization coordinate. Ions originating beyond the point $z=3.4 \mathrm{~cm}$ do not reach the cathode.

\section{B. Motion of ions off axis}

To calculate the radial displacement of ion trajectories at the cathode, we numerically solved equation (23). The beam field was not included in these calculations. To solve equation (23) we wrote a short $\mathrm{C}++$ code using the $1^{\text {st }}$ order Euler method. Higher order integration methods were not needed in this case because ion trajectories were smooth and the curvature of the trajectories was small. The equation of motion was integrated with the time step equal to $6^{\circ}$ of the RF phase. The red curve in Figure 6 shows the deviation of ion trajectories from the initial radius calculated at the cathode and normalized to the initial radius vs. the ionization coordinate. According to the result, the radius of ion trajectories at the cathode does not exceed the initial radius by more than $17 \%$ for almost all ions that originate between 0 and 3.4 $\mathrm{cm}$.

Also, Figure 6 shows the deviation of ion trajectories at the cathode given by analytical expression (29). The mathematical package Mathcad [12] and the field on the gun axis (Fig. 2) were used to calculate the integrals in (29). This result is in a good agreement with the numerical solution of (23) described in previous paragraph up to $z \approx 3 \mathrm{~cm}$. Equation $(29)$ predicts a large orbit displacement for $z>3$ and, therefore, cannot be used for ions originating in that region.

The effect of the beam field on the transverse motion depends on the beam current. A pulsed electron beam with a bunch radius of $2 \mathrm{~mm}$ and a bunch rep rate of $9.38 \mathrm{MHz}$ will have a net focusing effect on $\mathrm{H}_{2}^{+}$ions if the beam current is below $500 \mathrm{~mA}$. As the maximum beam current will not exceed $50 \mathrm{~mA}$ in the $9.38 \mathrm{MHz}$ operational mode, it is safe to assume that almost all ions originating between 0 and $3.4 \mathrm{~cm}$ along the gun axis will strike the cathode.

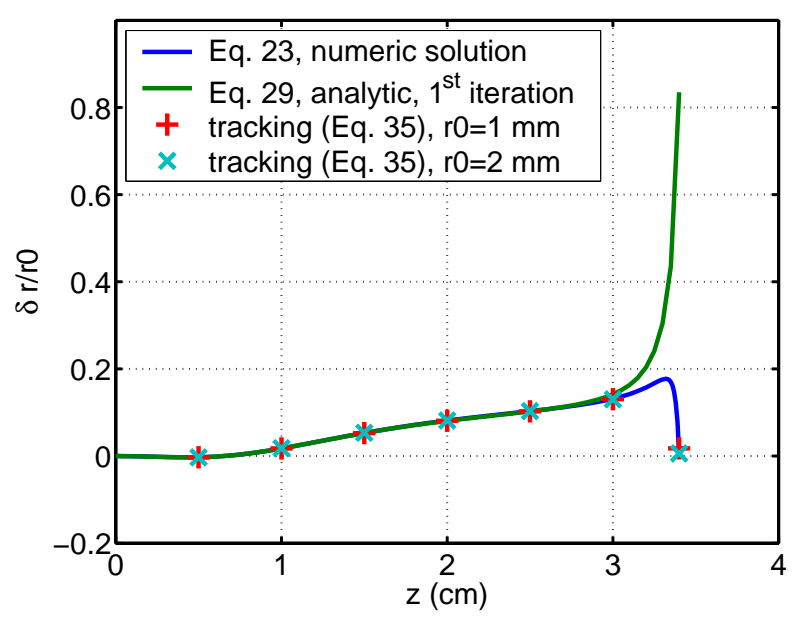

FIG. 6: Normalized deviation of ion trajectories from the initial radius at the cathode as a function of the ionization coordinate. Tracking results for $r_{0}=1 \mathrm{~mm}$ and $r_{0}=2 \mathrm{~mm}$ practically coincide with each other that confirms linearity of the ion motion close to the gun axis.

\section{Validation by tracking}

To test the results described above a short $\mathrm{C}++$ tracking code was developed. The code calculated ion trajectories in two-dimensional RF field maps generated by $\mathrm{Su}-$ perFish. The field of the electron beam was not included in these simulations. Using the classical $4^{\text {th }}$ order RungeKutta integration method, the code solved the following system of equations:

$$
\begin{aligned}
\frac{\mathrm{d} z}{\mathrm{~d} \phi} & =\tilde{p}_{z} \\
\frac{\mathrm{d} \tilde{p}_{z}}{\mathrm{~d} \phi} & =\frac{Z e E_{z}}{A m_{u} c^{2}} \lambda^{2} \\
\frac{\mathrm{d} r}{\mathrm{~d} \phi} & =\tilde{p}_{r} \\
\frac{\mathrm{d} \tilde{p}_{z}}{\mathrm{~d} \phi} & =\frac{Z e}{A m_{u} c^{2}}\left(E_{r} \lambda-\tilde{p}_{z} B_{\phi}\right) \lambda
\end{aligned}
$$

where $\phi$ is the RF phase used as the independent variable. $\tilde{p}_{r, z}$ is related to the particle momentum $p_{r, z}$ as

$$
\tilde{p}_{r, z}=\frac{p_{r, z}}{m \omega},
$$

where $\omega$ is the RF angular frequency. Note that the magnetic field term cannot be neglected in (35d) as its effect on the averaged radial motion of ions is comparable to the effect of inhomogenuity of the electric field (see (6)). Although it seemingly contradicts equation (10), it is trivial to show that the term $\nabla \mathcal{E}^{2}$ in (10) includes the magnetic force.

In simulating the beam dynamics in the BNL gun, test particles were launched from nodes of a rectangular $r-z$ mesh. The starting points were uniformly distributed over the interval $z=0.5-10 \mathrm{~cm}$ with a step of $5 \mathrm{~mm}$. 
Radially, the nodes were located at $r=0,1$ and $2 \mathrm{~mm}$. The simulated axial motion agreed very well with predictions of the 1D model described in Section III A: Ions originating between the cathode and $z \approx 3.4 \mathrm{~cm}$ reached the cathode while ions originating at $z>3.4 \mathrm{~cm}$ left the gun. The result of simulation of the radial dynamics is shown in Figure 6. The figure shows the simulated radial deviation of ion trajectories from the initial radius at the cathode normalized to the initial radius. The initial radius of trajectories $r_{0}$ was equal to $1 \mathrm{~mm}$ and 2 $\mathrm{mm}$. As expected, the tracking result is in a very good agreement with the numerical solution of (23). Also, the tracking result agrees well with (29) for $z \leq 3 \mathrm{~cm}$. As mentioned earlier, equation (29) fails to describe the ion motion correctly if ions originate in the region $z>3 \mathrm{~cm}$ that explains the discrepancy between the simulation and analytical results in that region. Also, note that trajectories corresponding to different initial radii normalized to the initial radius yield almost the same result that proves linearity of the radial ion motion.

\section{Rate of ion bombardment. Comparison to a DC gun.}

The number of ions bombarding the cathode normalized to the extracted charge is given by

$$
\frac{\mathrm{d} N}{\mathrm{~d} Q}=\frac{n_{i}}{e} \int_{0}^{D} \sigma(E(z)) \mathrm{d} z,
$$

where $n_{i}$ is the particle density of the residual gas, $\sigma$ is the ionization cross section, and $D$ is the distance from which ions can reach the cathode. Figure 7 shows the $\mathrm{H}_{2}$ ionization cross section as a function of the electron beam energy [13]. For the BNL gun, we assumed a residual hydrogen pressure of $5 \cdot 10^{-12}$ Torr and the distance $D$ equal to $3.4 \mathrm{~cm}$. The beam energy as a function of $z$ was calculated by Parmela. For these parameters, equation (37) yielded

$$
\left(\frac{d N}{d Q}\right)_{\mathrm{BNL} \mathrm{SRF}}=1.7 \cdot 10^{6} \text { ions } / \mathrm{C} .
$$

According to Sections IV A and IV B, almost all these ions will strike the cathode.

This number can be compared to the number of ions produced in a high voltage (HV) DC gun. For this example, we have chosen the following parameters: the beam energy was $650 \mathrm{keV}$, the accelerating gap was $5 \mathrm{~cm}$, and the residual gas pressure was the same as that in the BNL gun example, $5 \cdot 10^{-12}$ Torr. For these parameters, equation (37) yielded

$$
\left(\frac{d N}{d Q}\right)_{\mathrm{HV} \mathrm{DC}}=2.4 \cdot 10^{6} \mathrm{ions} / \mathrm{C} .
$$

In principle, ions produced in a transfer line can be trapped in the beam and travel towards a gun as described in [14]. It is obvious that low energy trapped

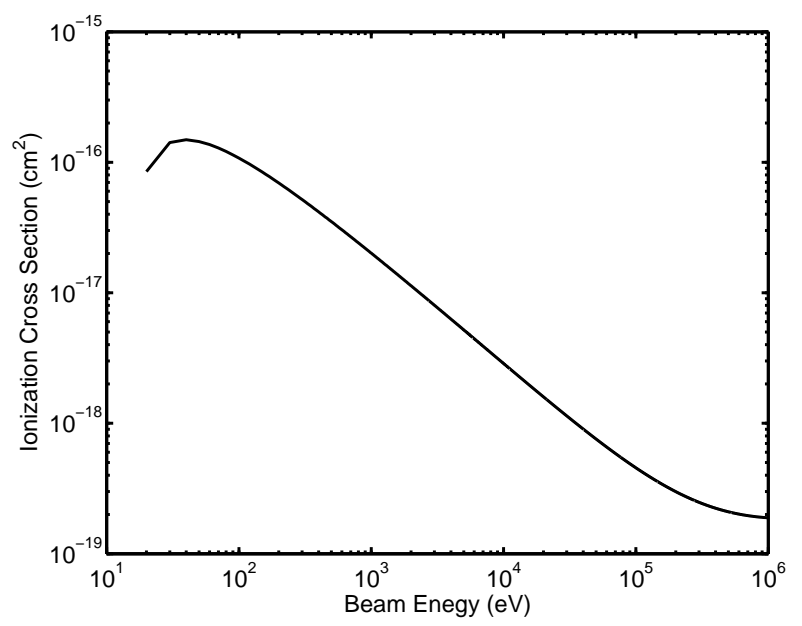

FIG. 7: $\mathrm{H}_{2}$ ionization cross section as a function of the electron beam energy.

ions cannot reach the cathode in the BNL gun because of the effective potential barrier produced by the accelerating RF field. In a DC gun, the flux of trapped ions can be eliminated by means of biasing the anode to a positive potential of a few hundred or thousand Volts as proposed in [14].

Results (38) and (39) predict similar rates of ion bombardment in the BNL gun and in the DC example, indicating that ion bombardment can affect the cathode lifetime in the BNL gun. However, it is difficult to make an accurate cross-comparison of the cathode lifetime between DC and RF guns based on the over-all rate of cathode bombardment. First, the ion energy spectra in RF and DC guns are substantially different. Second, DC guns are frequently operated with the laser spot shifted from the cathode center that causes a fraction of high energy ions to miss the laser spot. Therefore, detailed knowledge of ion spectra along with QE damage efficiency as a function of the ion energy are required to predict the cathode lifetime with a reasonable accuracy.

In the end, we would like to point out that a large portion of ions are produced within a distance of a few millimeters from the cathode. Figure 8 shows the fraction of ions produced within the interval between 0 and $z$ as a function of $z$ for the BNL gun and the HV DC example described above. According to this result, approximately a half of all ions that can reach the cathode come from a distance of $2-3 \mathrm{~mm}$. Because of this, improving vacuum is the only currently available efficient way of suppressing ion bombardment in DC guns. In RF guns, in addition to vacuum improvement, cathode bombardment can be suppressed by a proper choice of the accelerating phase as described in Section III A. For the BNL gun, this technique can reduce the rate of cathode bombardment by an additional factor of 5 or so. 


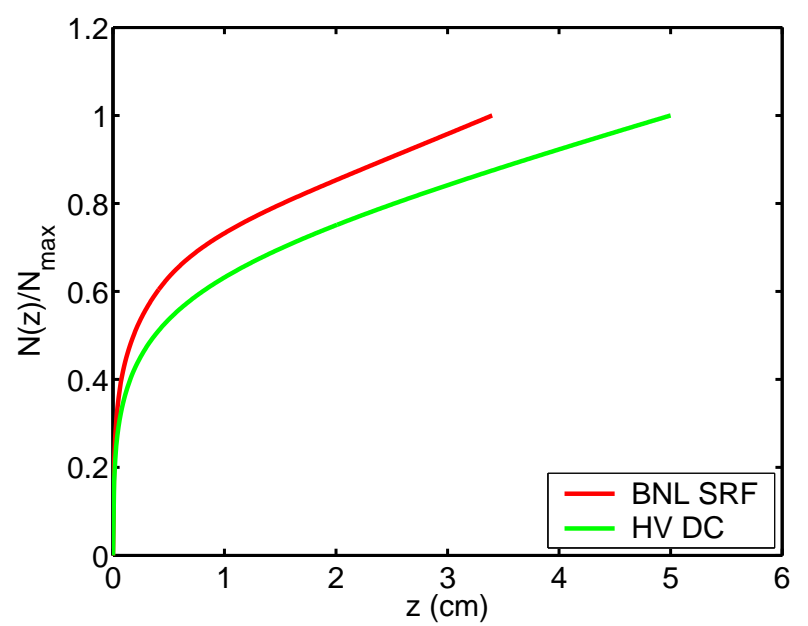

FIG. 8: Fraction of ions reaching the cathode as a function of $z$.

[1] A.V.Aleksandrov, N.S.Dikansky, R.G.Gromov, and P.V.Logatchov, in Proceedings of PAC 1999, New York, New York, 1999 (IEEE, Piscataway, New Jersey, 1999), p. 78.

[2] R. Fliller, T. Anderson, H. Edwards, H. Bluem, T. Schultheiss, C. Sinclair, and M. Huening, in Proceedings of PAC 2005, Knoxville, Tennessee, 2005 (IEEE, Piscataway, New Jersey, 2005), p. 2708.

[3] J. Kewisch, I. Ben-Zvi, T. Rao, A. Burrill, D. Pate, R. Grover, R. Todd, H. Bluem, D. Holmes, and T. Schultheiss, in Proceedings of the Workshop on Polarized Sources, Targets and Polarimetry - PSTP 2007, BNL, Upton, New York, 2007 (AIP, Melville, New York, 2007), p. 118.

[4] J. W. Lewellen, Phys. Rev. ST Accel. Beams 5, 020101 (2002).

[5] P. L. Kapitza, Zh. Eksp. Teor. Fiz. 21 (1951).

[6] A. V. Gaponov and M. A. Miller, Zh. Eksp. Teor. Fiz. 34 (1958), [Sov. Phys. JETP 7, 168 (1958)].

[7] A. Poncet, in Proceedings of CERN Accelerator School: 5th Advanced Accelerator Physics Course, Rhodes, Greece, 1993 (CERN, 1995), p. 859.

\section{CONCLUSIONS}

The method of rapidly oscillating field provides insight into the motion of ions in RF guns without employing tracking. Using the method, one can find the rate of cathode bombardment and the impact energy of ions as functions of the RF frequency, accelerating gradient, and accelerating phase. With this information, one can develop mitigation techniques similar to those described in the paper in order to reduce the rate of ion backbombardment.

\section{Acknowledgments}

The authors would like to thank Ilan Ben-Zvi for bringing up the subject of ion bombardment in RF guns to their attention.
[8] K. Halbach and R. Holsinger, Particle Accelerators 7, 21 (1976).

[9] R. Calaga, I. Ben-Zvi, M. Blaskiewicz, X. Chang, D. Kayran, and V. Litvinenko, Physica C 441, 159 (2006).

[10] V. Litvinenko, D. Beavis, I. Ben-Zvi, M. Blaskiewicz, A. Burrill, R. Calaga, P. Cameron, X. Chang, A. Drees, G. Ganetis, et al., in Proceedings of EPAC08, Genoa, Italy, 2008 (EPS-AG/CERN, Geneva, 2008), p. 193, URL http://accelconf .web.cern.ch/AccelConf/e08/ papers/mopc057.pdf.

[11] L. Young, Tech. Rep. LA-UR-96-1835, LANL (1996), documentation by J.H. Billen, rev. 2004.

[12] Mathcad, PTC, PTC Corporate Headquarters, 140 Kendrick Street, Needham, MA 02494, USA (2008), URL http://www.ptc.com/products/mathcad/.

[13] M. Reiser, Theory and Design of Charged Particle Beams (John Wiley \& Sons, Inc., 1994).

[14] E. Pozdeyev, Phys. Rev. ST Accel. Beams 10, 083501 (2007). 